\title{
771.
}

\section{SPECIMEN OF A LITERAL TABLE FOR BINARY QUANTICS, OTHERWISE A PARTITION TABLE.}

[From the American Journal of Mathematics, vol. Iv. (1881), pp. 248-255.]

The Table, commencing $1 ; b ; c, b^{2} ; d, b c, b^{3} ; \ldots$, is in fact a Partition Table, viz. considering the letters $b, c, d, \ldots$ as denoting $1,2,3, \ldots$ respectively, it is $1^{\circ}$; $1 ; 2,11 ; 3,12,111 ; \ldots$ a table of the partitions of the numbers $0,1,2,3, \ldots$, expressed however in the literal form, in order to its giving the literal terms which enter into the coefficients of any covariant of a binary quantic. The table ought to have been made and published many years ago, before the calculation of the covariants of the quintic; and the present publication of it is, in some measure, an anachronism: but $I$ in fact felt the need of it in some calculations in regard to the sextic; and I think the table may be found useful on other occasions. I have contented myself with calculating the table up to $s=18$, that is, so as to include in it all the partitions of 18: it would, I think, be desirable to extend it further, say to $z=26$; or even beyond this point, but perhaps without introducing any new letters, (that is, so as to give for the higher numbers only the partitions with a largest part not exceeding 26): the question of the space which such a table would occupy will be considered presently.

As to the employment of the table, observe that, in applying it to the case of a quantic $(a, b, c, d \gamma x, y)^{3}$, the terms containing the letters $e, f$, etc., posterior to the last coefficient $d$ of the quantic are to be disregarded; and that the terms are to be rendered homogeneous by the introduction of the proper power of the first coefficient $a$, rejecting any term for which the exponent of $a$ would be negative (or what is the same thing, any term of too high a degree in the coefficients $b, c, d)$; 
thus, for the cubicovariant, where the coefficients are of the degree 3 , and of the weights $3,4,5,6$ respectively, from the portion of the table

$\begin{array}{llll}d & e & f & g \\ b c & b d & b e & b f \\ b^{3} & c^{2} & c d & c e \\ & b^{2} c & b^{2} d & d^{2} \\ & b^{4} & b c^{2} & b^{2} e \\ & & b^{3} c & b c d \\ & b^{5} & c^{3} \\ & & b^{3} d \\ & & & \text { etc. }\end{array}$

we at once copy out the terms

\begin{tabular}{l|l|l|l}
$a^{2} d$ & $a b d$ & $a c d$ & $a d^{2}$ \\
$a b c$ & $a c^{2}$ & $b^{2} d$ & $b c d$ \\
$b^{3}$ & $b^{2} c$ & $b c^{2}$ & $c^{3}$
\end{tabular}

which compose the coefficients in question.

As regards the formation of the table, this is at once effected, and the successive terms are obtained currente calamo, by Arbogast's rule of the last and the last but one: observing that each term is to be regarded as containing implicitly a power of $a$, so that operating on any term such as $b^{4}$, the operation on the last letter gives $b^{3} c$, and that on the last but one letter gives $b^{5}$. There is little risk of error except in the accidental omission of a term; but of course any one omission would occasion the omission of all the subsequent terms derivable from the omitted term, and would so be fatal: to remove this source of error, observe that for the successive numbers $0,1,2,3$, etc., the number of partitions should be

\begin{tabular}{cccccccccccccccccccc}
0 & 1 & 2 & 3 & 4 & 5 & 6 & 7 & 8 & 9 & 10 & 11 & 12 & 13 & 14 & 15 & 16 & 17 & 18 & $\ldots$ \\
\hline 1 & 1 & 2 & 3 & 5 & 7 & 11 & 15 & 22 & 30 & 42 & 56 & 77 & 101 & 135 & 176 & 231 & 297 & 385 & $\ldots$
\end{tabular}

and we can thus, for each partible number successively, verify that the right number of partitions has been obtained.

But as the number of partitions becomes large, a further control is convenient, and even necessary-say we have the 176 partitions of 15 , we have by the rule to derive thence the 231 partitions of 16 , and it is not until the whole of this derivation is gone through, that we could by counting the number of the new terms ascertain that the right number of 231 terms has been obtained. To break up the verification, it is convenient to know that for the partitions of 16 into 1 part, 2 parts, 3 parts, 4 parts, etc., the numbers of partitions are 1, 8, 21, 34, etc., respectively: we can then as soon as the derivations giving the partitions into 1 part, 2 parts, 3 parts, etc., respectively, have been performed, verify that the right numbers $1,8,21,34$, etc., of terms have been obtained. The numbers are contained in the following table, each column of which is calculated from the preceding columns according to a rule which 
is easily obtained, and which is itself verified by the condition that the sums of the numbers in the several columns give the before mentioned series of numbers 1 , $1,2,3,5,7$, etc.

No. of

Partible Number.

\begin{tabular}{l|lllllllllllllllllll} 
Parts. & 0 & 1 & 2 & 3 & 4 & 5 & 6 & 7 & 8 & 9 & 10 & 11 & 12 & 13 & 14 & 15 & 16 & 17 & 18
\end{tabular}

\begin{tabular}{|l|l|l|l|l|l|l|l|l|l|l|l|l|l|l|l|l|l|l|}
1 & 1 \\
2
\end{tabular}

The practical rule for the construction of the table thus is:-On a sheet of paper ruled in squares, and which is read as a continuous column from the bottom of one column to the top of the next column, form the terms by Arbogast's method as already explained; writing down in pencil a batch of terms, and counting them 
to see that the right number has been obtained, then, at the same time verifying the derivations, mark these over in ink; and so on with another batch of terms, until the whole number of the partitions of any particular number is obtained.

The foregoing series $1,1,2,3, \ldots, 385$, for the number of the partitions of the successive numbers $0,1,2,3, \ldots, 18$ is carried by Euler up to the number of partitions of $59,=831820$, see the paper "De Partitione Numerorum," Op. Arith. Coll. I., bottom line of the table pp. 97-101: the continuation from the number 385 and for the partible numbers 19 to 30 is as follows:

\begin{tabular}{cccccccccccc}
19 & 20 & 21 & 22 & 23 & 24 & 25 & 26 & 27 & 28 & 29 & 30 \\
\hline 490 & 627 & 792 & 1002 & 1255 & 1575 & 1958 & 2436 & 3010 & 3718 & 4565 & 5604
\end{tabular}

the whole number of terms $1,1, \ldots, 5604$ amounts to 28629 , which at the rate of 500 to a page would occupy somewhat under 60 pages; or, at the rate here employed of 369 to a page, somewhat under 78 pages.

The Partition Table, 0 to 18.

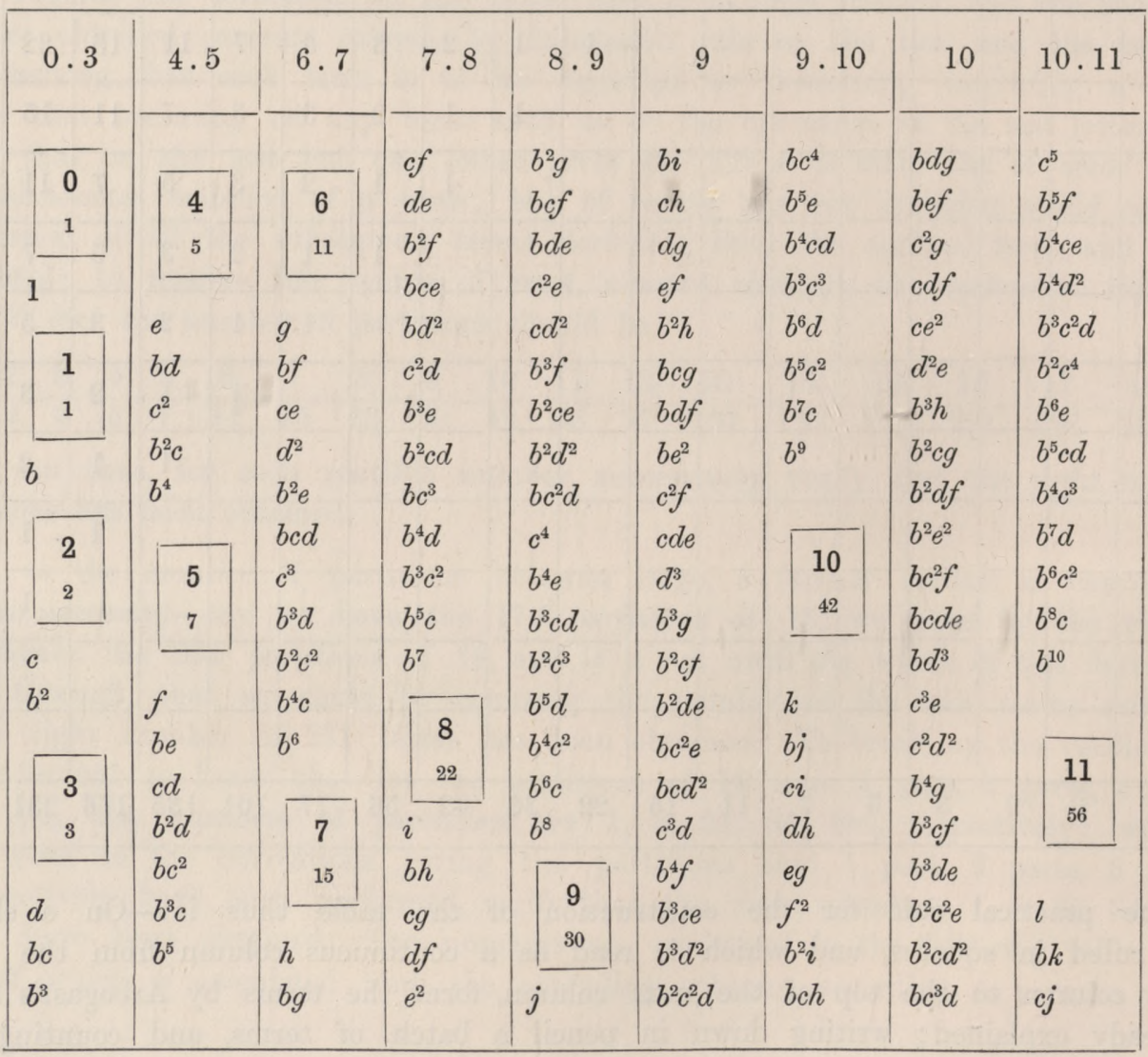


The Partition Table, 0 to 18 (continued).

\begin{tabular}{|c|c|c|c|c|c|c|c|c|}
\hline 11 & 11.12 & 12 & 12.13 & 13 & 13.14 & 14 & 14 & 14 \\
\hline$d i$ & $b^{6} f$ & bcef & $b^{6} d^{2}$ & bceg & $b c^{3} d^{2}$ & $g i$ & $b^{4} k$ & $c^{5} e$ \\
\hline$e h$ & $b^{5} c e$ & $b d^{2} f$ & $b^{5} c^{2} d$ & $b c f^{2}$ & $c^{5} d$ & $h^{2}$ & $b^{3} c j$ & $c^{4} d^{2}$ \\
\hline$f g$ & $b^{5} d^{2}$ & $b d e^{2}$ & $b^{4} c^{4}$ & $b d^{2} g$ & $b^{6} h$ & $b^{2} m$ & $b^{3} d i$ & $b^{6} i$ \\
\hline$b^{2} j$ & $b^{4} c^{2} d$ & $c^{3} g$ & $b^{8} e$ & bdef & $b^{5} \mathrm{cg}$ & $b c l$ & $b^{3} e h$ & $b^{5} c h$ \\
\hline$b c i$ & $b^{3} c^{4}$ & $c^{2} d f$ & $b^{7} c d$ & $b e^{3}$ & $b^{5} d f$ & $b d k$ & $b^{3} f g$ & $b^{5} d g$ \\
\hline$b d h$ & $b^{7} e$ & $c^{2} e^{2}$ & $b^{6} c^{3}$ & $c^{3} h$ & $b^{5} e^{2}$ & $b e j$ & $b^{2} c^{2} i$ & $b^{5} e f$ \\
\hline beg & $b^{6} c d$ & $c d^{2} e$ & $b^{9} d$ & $c^{2} d g$ & $b^{4} c^{2} f$ & $b f i$ & $b^{2} c d h$ & $b^{4} c^{2} g$ \\
\hline$b f^{2}$ & $b^{5} c^{3}$ & $d^{4}$ & $b^{8} c^{2}$ & $c^{2} e f$ & $b^{4} c d e$ & $b g h$ & $b^{2} c e g$ & $b^{4} c d f$ \\
\hline$c^{2} h$ & $b^{8} d$ & $b^{4} i$ & $b^{10} c$ & $c d^{2} f$ & $b^{4} d^{3}$ & $c^{2} k$ & $b^{2} c f^{2}$ & $b^{4} c e^{2}$ \\
\hline$c d g$ & $b^{7} c^{2}$ & $b^{3} c h$ & $b^{12}$ & $c d e^{2}$ & $b^{3} c^{3} e$ & $c d j$ & $b^{2} d^{2} g$ & $b^{4} d^{2} e$ \\
\hline$c e f$ & $b^{9} c$ & $b^{3} d g$ & & $d^{3} e$ & $b^{3} c^{2} d^{2}$ & $c e i$ & $b^{2} d e f$ & $b^{3} c^{3} f$ \\
\hline$d^{2} f$ & $b^{11}$ & $b^{3} e f$ & 13 & $b^{4} j$ & $b^{2} c^{4} d$ & $c f h$ & $b^{2} e^{3}$ & $b^{3} c^{2} d e$ \\
\hline$d e^{2}$ & & $b^{2} c^{2} g$ & 101 & $b^{3} c i$ & $b c^{6}$ & $c g^{2}$ & $b c^{3} h$ & $b^{3} c d^{3}$ \\
\hline$b^{3} i$ & 12 & $b^{2} c d f$ & $n$ & $b^{3} d h$ & $b^{7} g$ & $d^{2} i$ & $b c^{2} d g$ & $b^{2} c^{4} e$ \\
\hline$b^{2} c h$ & 77 & $b^{2} c e^{2}$ & $b m$ & $b^{3} e g$ & $b^{6} c f$ & deh & $b c^{2} e f$ & $b^{2} c^{3} d^{2}$ \\
\hline$b^{2} d g$ & $m$ & $b^{2} d^{2} e$ & $c l$ & $b^{3} f^{2}$ & $b^{6} \mathrm{de}$ & $d f g$ & $b c d^{2} f$ & $b c^{5} d$ \\
\hline$b^{2} e f$ & $b l$ & $b c^{3} f$ & $d k$ & $b^{2} c^{2} h$ & $b^{5} c^{2} e$ & $e^{2} g$ & $b c d e^{2}$ & $c^{7}$ \\
\hline$b c^{2} g$ & $c k$ & $b c^{2} d e$ & $e j$ & $b^{2} c d g$ & $b^{5} c d^{2}$ & $e f^{2}$ & $b d^{3} e$ & $b^{7} h$ \\
\hline$b c d f$ & $d j$ & $b c d^{3}$ & $f i$ & $b^{2} c e f$ & $b^{4} c^{3} d$ & $b^{3} l$ & $c^{4} g$ & $b^{6} \mathrm{cg}$ \\
\hline$b c e^{2}$ & $e i$ & $c^{4} e$ & $g h$ & $b^{2} d^{2} f$ & $b^{3} c^{5}$ & $b^{2} c k$ & $c^{3} d f$ & $b^{6} d f$ \\
\hline$b d^{2} e$ & $f h$ & $c^{3} d^{2}$ & $b^{2} l$ & $b^{2} d e^{2}$ & $b^{8} f$ & $b^{2} d j$ & $c^{3} e^{2}$ & $b^{6} e^{2}$ \\
\hline$c^{3} f$ & $g^{2}$ & $b^{5} h$ & $b c k$ & $b c^{3} g$ & $b^{7} c e$ & $b^{2} e i$ & $c^{2} d^{2} e$ & $b^{5} c^{2} f$ \\
\hline$c^{2} d e$ & $b^{2} k$ & $b^{4} c g$ & $b d j$ & $b c^{2} d f$ & $b^{7} d^{2}$ & $b^{2} f h$ & $c d^{4}$ & $b^{5} c d e$ \\
\hline$c d^{3}$ & $b c j$ & $b^{4} d f$ & $b e i$ & $b c^{2} e^{2}$ & $b^{6} c^{2} d$ & $b^{2} g^{2}$ & $b^{5} j$ & $b^{5} d^{3}$ \\
\hline$b^{4} h$ & $b d i$ & $b^{4} e^{2}$ & $b f h$ & $b c d^{2} e$ & $b^{5} c^{4}$ & $b c^{2} j$ & $b^{4} c i$ & $b^{4} c^{3} e$ \\
\hline$b^{3} c g$ & beh & $b^{3} c^{2} f$ & $b g^{2}$ & $b d^{4}$ & $b^{9} e$ & $b c d i$ & $b^{4} d h$ & $b^{4} c^{2} d^{2}$ \\
\hline$b^{3} d f$ & $b f g$ & $b^{3} c d e$ & $c^{2} j$ & $c^{4} f$ & $b^{8} c d$ & bceh & $b^{4} e g$ & $b^{3} c^{4} d$ \\
\hline$b^{3} e^{2}$ & $c^{2} i$ & $b^{3} d^{3}$ & $c d i$ & $c^{3} d e$ & $b^{7} c^{3}$ & $b c f g$ & $b^{4} f^{2}$ & $b^{2} c^{6}$ \\
\hline$b^{2} c^{2} f$ & $c d h$ & $b^{2} c e^{2}$ & $c e h$ & $c^{2} d^{3}$ & $b^{10} d$ & $b d^{2} h$ & $b^{3} c^{2} h$ & $b^{8} g$ \\
\hline$b^{2} c d e$ & $c e g$ & $b^{2} c^{2} d^{2}$ & $c f g$ & $b^{5} i$ & $b^{9} c^{2}$ & bdeg & $b^{3} c d g$ & $b^{7} c f$ \\
\hline$b^{2} d^{3}$ & $c f^{2}$ & $b c^{4} d$ & $d^{2} h$ & $b^{4} c h$ & $b^{11} c$ & $b d f^{2}$ & $b^{3} c e f$ & $b^{7} d e$ \\
\hline$b c^{3} e$ & $d^{2} g$ & $c^{6}$ & deg & $b^{4} d g$ & $b^{13}$ & $b e^{2} f$ & $b^{3} d^{2} f$ & $b^{6} c^{2} e$ \\
\hline$b c^{2} d^{2}$ & $\operatorname{def}$ & $b^{6} g$ & $d f^{2}$ & $b^{4} e f$ & & $c^{3} i$ & $b^{3} d e^{2}$ & $b^{6} c d^{2}$ \\
\hline$c^{4} d$ & $e^{3}$ & $b^{5} c f$ & $e^{2} f$ & $b^{3} c^{2} g$ & 14 & $c^{2} d h$ & $b^{2} c^{3} g$ & $b^{5} c^{3} d$ \\
\hline$b^{5} g$ & $b^{3} j$ & $b^{5} d e$ & $b^{3} k$ & $b^{3} c d f$ & 135 & $c^{2} e g$ & $b^{2} c^{2} d f$ & $b^{4} c^{5}$ \\
\hline$b^{4} c f$ & $b^{2} c i$ & $b^{4} c^{2} e$ & $b^{2} c j$ & $b^{3} c e^{2}$ & 0 & $c^{2} f^{2}$ & $b^{2} c^{2} e^{2}$ & $b^{9} f$ \\
\hline$b^{4} \mathrm{de}$ & $b^{2} d h$ & $b^{4} c d^{2}$ & $b^{2} d i$ & $b^{3} d^{2} e$ & $b n$ & $c d^{2} g$ & $b^{2} c d^{2} e$ & $b^{8} c e$ \\
\hline$b^{3} c^{2} e$ & $b^{2} e g$ & $b^{3} c^{3} d$ & $b^{2} e h$ & $b^{2} c^{3} f$ & $\mathrm{~cm}$ & cdef & $b^{2} d^{4}$ & $b^{8} d^{2}$ \\
\hline$b^{3} c d^{2}$ & $b^{2} f^{2}$ & $b^{2} c^{5}$ & $b^{2} f g$ & $b^{2} c^{2} d e$ & $d l$ & $c e^{3}$ & $b c^{4} f$ & $b^{7} c^{2} d$ \\
\hline$b^{2} c^{3} d$ & $b c^{2} h$ & $b^{7} f$ & $b c^{2} i$ & $b^{2} c d^{3}$ & $e k$ & $d^{3} f$ & $b e^{3} d e$ & $b^{6} c^{4}$ \\
\hline$b c^{5}$ & $b c d g$ & $b^{6} c e$ & $b c d h$ & $b c^{4} e$ & $f j$ & $d^{2} e^{2}$ & $b c^{2} d^{3}$ & $b^{10} e$ \\
\hline
\end{tabular}

C. XI. 
The Partition Table, 0 to 18 (continued).

\begin{tabular}{|c|c|c|c|c|c|c|c|c|}
\hline 14.15 & 15 & 15 & 15 & 15.16 & 16 & 16 & 16 & 16 \\
\hline$b^{9} c d$ & $b^{2} g h$ & $b c d e f$ & $b^{5} f^{2}$ & $b^{4} c^{4} d$ & $c d l$ & $c e f^{2}$ & $c d^{2} e^{2}$ & $b^{5} f g$ \\
\hline$b^{8} c^{3}$ & $b c^{2} k$ & $b c e^{3}$ & $b^{4} c^{2} h$ & $b^{3} c^{6}$ & cek & $d^{3} h$ & $d^{4} e$ & $b^{4} c^{2} i$ \\
\hline$b^{11} d$ & $b c d j$ & $b d^{3} f$ & $b^{4} c d g$ & $b^{9} g$ & $c f j$ & $d^{2} e g$ & $b^{5} l$ & $b^{4} c d h$ \\
\hline$b^{10} c^{2}$ & $b c e i$ & $b d^{2} e^{2}$ & $b^{4} c e f$ & $b^{8} c f$ & $c g i$ & $d^{2} f^{2}$ & $b^{4} c k$ & $b^{4} c e g$ \\
\hline$b^{12} c$ & $b c f h$ & $c^{4} h$ & $b^{4} d^{2} f$ & $b^{8} d e$ & $c h^{2}$ & $d e^{2} f$ & $b^{4} d j$ & $b^{4} c f^{2}$ \\
\hline$b^{14}$ & $b c g^{2}$ & $c^{3} d g$ & $b^{4} d e^{2}$ & $b^{7} c^{2} e$ & $d^{2} k$ & $e^{4}$ & $b^{4} e i$ & $b^{4} d^{2} g$ \\
\hline & $b d^{2} i$ & $c^{3} e f$ & $b^{3} c^{3} g$ & $b^{7} c d^{2}$ & $d e j$ & $b^{4} m$ & $b^{4} f h$ & $b^{4} d e f$ \\
\hline 15 & bdeh & $c^{2} d^{2} f$ & $b^{3} c^{2} d f$ & $b^{6} c^{3} d$ & $d f i$ & $b^{3} c l$ & $b^{4} g^{2}$ & $b^{4} e^{3}$ \\
\hline 176 & $b d f g$ & $c^{2} d e^{2}$ & $b^{3} c^{2} e^{2}$ & $b^{5} c^{5}$ & $d g h$ & $b^{3} d k$ & $b^{3} c^{2} j$ & $b^{3} c^{3} h$ \\
\hline$p$ & $b e^{2} g$ & $c d^{3} e$ & $b^{3} c d^{2} e$ & $b^{10} f$ & $e^{2} i$ & $b^{3} e j$ & $b^{3} c d i$ & $b^{3} c^{2} d g$ \\
\hline bo & $b e f^{2}$ & $d^{5}$ & $b^{3} d^{4}$ & $b^{9} c e$ & efh & $b^{3} f i$ & $b^{3} c e h$ & $b^{3} c^{2} e f$ \\
\hline$c n$ & $c^{3} j$ & $b^{5} k$ & $b^{2} c^{4} f$ & $b^{9} d^{2}$ & $e g^{2}$ & $b^{3} g h$ & $b^{3} c f g$ & $b^{3} c d^{2} f$ \\
\hline$d m$ & $c^{2} d i$ & $b^{4} c j$ & $b^{2} c^{3} d e$ & $b^{8} c^{2} d$ & $f^{2} g$ & $b^{2} c^{2} k$ & $b^{3} d^{2} h$ & $b^{3} c d e^{2}$ \\
\hline$e l$ & $c^{2} e h$ & $b^{4} d i$ & $b^{2} c^{2} d^{3}$ & $b^{7} c^{4}$ & $b^{3} n$ & $b^{2} c d j$ & $b^{3} \mathrm{deg}$ & $b^{3} d^{3} e$ \\
\hline$f k$ & $c^{2} f g$ & $b^{4} e h$ & $b c^{5} e$ & $b^{11} e$ & $b^{2} \mathrm{~cm}$ & $b^{2} c e i$ & $b^{3} d f^{2}$ & $b^{2} c^{4} g$ \\
\hline$g j$ & $c d^{2} h$ & $b^{4} f g$ & $b c^{4} d^{2}$ & $b^{10} c d$ & $b^{2} d l$ & $b^{2} c f h$ & $b^{3} e^{2} f$ & $b^{2} c^{3} d f$ \\
\hline$h i$ & $c d e g$ & $b^{3} c^{2} i$ & $c^{6} d$ & $b^{9} c^{3}$ & $b^{2} e k$ & $b^{2} c g^{2}$ & $b^{2} c^{3} i$ & $b^{2} c^{3} e^{2}$ \\
\hline$b^{2} n$ & $c d f^{2}$ & $b^{3} c d h$ & $b^{7} i$ & $b^{12} d$ & $b^{2} f j$ & $b^{2} d^{2} i$ & $b^{2} c^{2} d h$ & $b^{2} c^{2} d^{2} e$ \\
\hline $\mathrm{bcm}$ & $c e^{2} f$ & $b^{3} c e g$ & $b^{6} c h$ & $b^{11} c^{2}$ & $b^{2} g i$ & $b^{2} d e h$ & $b^{2} c^{2} e g$ & $b^{2} c d^{4}$ \\
\hline$b d l$ & $d^{3} g$ & $b^{3} c f^{2}$ & $b^{6} d g$ & $b^{13} c$ & $b^{2} h^{2}$ & $b^{2} d f g$ & $b^{2} c^{2} f^{2}$ & $b c^{5} f$ \\
\hline bek & $d^{2} e f$ & $b^{3} d^{2} g$ & $b^{6} e f$ & $b^{15}$ & $b c^{2} l$ & $b^{2} e^{2} g$ & $b^{2} c d^{2} g$ & $b c^{4} d e$ \\
\hline$b f j$ & $d e^{3}$ & $b^{3} d e f$ & $b^{5} c^{2} g$ & & $b c d k$ & $b^{2} e f^{2}$ & $b^{2} c d e f$ & $b c^{3} d^{3}$ \\
\hline$b g i$ & $b^{4} l$ & $b^{3} e^{3}$ & $b^{5} c d f$ & 16 & $b c e j$ & $b c^{3} j$ & $b^{2} c e^{3}$ & $c^{6} e$ \\
\hline$b h^{2}$ & $b^{3} c k$ & $b^{2} c^{3} h$ & $b^{5} c e^{2}$ & 231 & $b c f i$ & $b c^{2} d i$ & $b^{2} d^{3} f$ & $c^{5} d^{2}$ \\
\hline$c^{2} l$ & $b^{3} d j$ & $b^{2} c^{2} d g$ & $b^{5} d^{2} e$ & $q$ & $b c g h$ & $b c^{2} e h$ & $b^{2} d^{2} e^{2}$ & $b^{7} j$ \\
\hline$c d k$ & $b^{3} e i$ & $b^{2} c^{2} e f$ & $b^{4} c^{3} f$ & $b p$ & $b d^{2} j$ & $b c^{2} f g$ & $b c^{4} h$ & $b^{6} c i$ \\
\hline$c e j$ & $b^{3} f h$ & $b^{2} c d^{2} f$ & $b^{4} c^{2} d e$ & co & $b d e i$ & $b c d^{2} h$ & $b c^{3} d g$ & $b^{6} d h$ \\
\hline$c f i$ & $b^{3} g^{2}$ & $b^{2} c d e^{2}$ & $b^{4} c d^{3}$ & $d n$ & $b d f h$ & bcdeg & $b c^{3} e f$ & $b^{6} e g$ \\
\hline$c g h$ & $b^{2} c^{2} j$ & $b^{2} d^{3} e$ & $b^{3} c^{4} e$ & $\mathrm{em}$ & $b d g^{2}$ & $b c d f^{2}$ & $b c^{2} d^{2} f$ & $b^{6} f^{2}$ \\
\hline$d^{2} j$ & $b^{2} c d i$ & $b c^{4} g$ & $b^{3} c^{3} d^{2}$ & $f l$ & $b e^{2} h$ & $b c e^{2} f$ & $b c^{2} d e^{2}$ & $b^{5} c^{2} h$ \\
\hline$d e i$ & $b^{2} c e h$ & $b c^{3} d f$ & $b^{2} c^{5} d$ & $g k$ & $b e f g$ & $b d^{3} g$ & $b c d^{3} e$ & $b^{5} c d g$ \\
\hline$d f h$ & $b^{2} c f g$ & $b c^{3} e^{2}$ & $b c^{7}$ & $h j$ & $b f^{3}$ & $b d^{2} e f$ & $b d^{5}$ & $b^{5} c e f$ \\
\hline$d g^{2}$ & $b^{2} d^{2} h$ & $b c^{2} d^{2} e$ & $b^{8} h$ & $i^{2}$ & $c^{3} k$ & $b d e^{3}$ & $c^{5} g$ & $b^{5} d^{2} f$ \\
\hline$e^{2} h$ & $b^{2} \mathrm{deg}$ & $b c d^{4}$ & $b^{7} c g$ & $b^{2} o$ & $c^{2} d j$ & $c^{4} i$ & $c^{4} d f$ & $b^{5} d e^{2}$ \\
\hline$e f g$ & $b^{2} d f^{2}$ & $c^{5} f$ & $b^{7} d f$ & $b c n$ & $c^{2} e i$ & $c^{3} d h$ & $c^{4} e^{2}$ & $b^{4} c^{3} g$ \\
\hline$f^{3}$ & $b^{2} e^{2} f$ & $c^{4} d e$ & $b^{7} e^{2}$ & $b d m$ & $c^{2} f h$ & $c^{3} e g$ & $c^{3} d^{2} e$ & $b^{4} c^{2} d f$ \\
\hline$b^{3} m$ & $b c^{3} i$ & $c^{3} d^{3}$ & $b^{6} c^{2} f$ & bel & $c^{2} g^{2}$ & $c^{3} f^{2}$ & $c^{2} d^{4}$ & $b^{4} c^{2} e^{2}$ \\
\hline$b^{2} c l$ & $b c^{2} d h$ & $b^{6} j$ & $b^{6} c d e$ & $b f k$ & $c d^{2} i$ & $c^{2} d^{2} g$ & $b^{6} k$ & $b^{4} c d^{2} e$ \\
\hline$b^{2} d k$ & $b c^{2} e g$ & $b^{5} c i$ & $b^{6} d^{3}$ & $b g j$ & cdeh & $c^{2} d e f$ & $b^{5} c j$ & $b^{4} d^{4}$ \\
\hline$b^{2} e j$ & $b c^{2} f^{2}$ & $b^{5} d h$ & $b^{5} c^{3} e$ & $b h i$ & $c d f g$ & $c^{2} e^{3}$ & $b^{5} d i$ & $b^{3} c^{4} f$ \\
\hline$b^{2} f i$ & $b c d^{2} g$ & $b^{5} e g$ & $b^{5} c^{2} d^{2}$ & $c^{2} m$ & $c e^{2} g$ & $c d^{3} f$ & $b^{5} e h$ & $b^{3} c^{3} d e$ \\
\hline
\end{tabular}


The Partition Table, 0 to 18 (continued).

\begin{tabular}{|c|c|c|c|c|c|c|c|c|}
\hline 16 & 16.17 & 17 & 17 & 17 & 17 & 17 & 17 & 17.18 \\
\hline$b^{3} c^{2} d^{3}$ & $b^{9} c^{2} d$ & ef $i$ & $d e f^{2}$ & $c^{2} d e g$ & $b c^{2} e^{3}$ & $b c^{5} g$ & $b^{7} f^{2}$ & $b^{7} c^{2} d^{2}$ \\
\hline$b^{2} c^{5} e$ & $b^{8} c^{4}$ & egh & $e^{3} f$ & $c^{2} d f^{2}$ & $b c d^{3} f$ & $b c^{4} d f$ & $b^{6} c^{2} h$ & $b^{6} c^{4} d$ \\
\hline$b^{2} c^{4} d^{2}$ & $b^{12} e$ & $f^{2} h$ & $b^{4} n$ & $c^{2} e^{2} f$ & $b c d^{2} e^{2}$ & $b c^{4} e^{2}$ & $b^{6} c d g$ & $b^{5} c^{6}$ \\
\hline$b c^{6} d$ & $b^{11} c d$ & $f g^{2}$ & $b^{3} \mathrm{~cm}$ & $c d^{3} g$ & $b d^{4} e$ & $b c^{3} d^{2} e$ & $b^{6} c e f$ & $b^{11} g$ \\
\hline$c^{8}$ & $b^{10} c^{3}$ & $b^{3} o$ & $b^{3} d l$ & $c d^{2} e f$ & $c^{5} h$ & $b c^{2} d^{4}$ & $b^{6} d^{2} f$ & $b^{10} c f$ \\
\hline$b^{8} i$ & $b^{13} d$ & $b^{2} c n$ & $b^{3} e k$ & $c d e^{3}$ & $c^{4} d g$ & $c^{6} f$ & $b^{6} d e^{2}$ & $b^{10} d e$ \\
\hline$b^{7} c h$ & $b^{12} c^{2}$ & $b^{2} d m$ & $b^{3} f j$ & $d^{4} f$ & $c^{4} e f$ & $c^{5} d e$ & $b^{5} c^{3} g$ & $b^{9} c^{2} e$ \\
\hline$b^{7} d g$ & $b^{14} c$ & $b^{2} e l$ & $b^{3} g i$ & $d^{3} e^{2}$ & $c^{3} d^{2} f$ & $c^{4} d^{3}$ & $b^{5} c^{2} d f$ & $b^{9} c d^{2}$ \\
\hline$b^{7} e f$ & $b^{16}$ & $b^{2} f k$ & $b^{3} h^{2}$ & $b^{5} m$ & $c^{3} d e^{2}$ & $b^{7} k$ & $b^{5} c^{2} e^{2}$ & $b^{8} c^{3} d$ \\
\hline$b^{6} c^{2} g$ & 17 & $b^{2} g j$ & $b^{2} c^{2} l$ & $b^{4} c l$ & $c^{2} d^{3} e$ & $b^{6} c j$ & $b^{5} c d^{2} e$ & $b^{7} c^{5}$ \\
\hline$b^{6} c d f$ & 17 & $b^{2} h i$ & $b^{2} c d k$ & $b^{4} d k$ & $c d^{5}$ & $b^{6} d i$ & $b^{5} d^{4}$ & $b^{12} f$ \\
\hline$b^{6} c e^{2}$ & 297 & $b c^{2} m$ & $b^{2} c e j$ & $b^{4} e j$ & $b^{6} l$ & $b^{6} e h$ & $b^{4} c^{4} f$ & $b^{11} c e$ \\
\hline$b^{6} d^{2} e$ & $r$ & $b c d l$ & $b^{2} c f i$ & $b^{4} f i$ & $b^{5} c k$ & $b^{6} f g$ & $b^{4} c^{3} d e$ & $b^{11} d^{2}$ \\
\hline$b^{5} c^{3} f$ & $b q$ & bcek & $b^{2} c g h$ & $b^{4} g h$ & $b^{5} d j$ & $b^{5} c^{2} i$ & $b^{4} c^{2} d^{3}$ & $b^{10} c^{2} d$ \\
\hline$b^{5} c^{2} d e$ & $c p$ & $b c f j$ & $b^{2} d^{2} j$ & $b^{3} c^{2} k$ & $b^{5} e i$ & $b^{5} c d h$ & $b^{3} c^{5} e$ & $b^{9} c^{4}$ \\
\hline$b^{5} c d^{3}$ & $d o$ & $b c g i$ & $b^{2} d e i$ & $b^{3} c d j$ & $b^{5} f h$ & $b^{5} c e g$ & $b^{3} c^{4} d^{2}$ & $b^{13} e$ \\
\hline$b^{4} c^{4} e$ & $e n$ & $b c h^{2}$ & $b^{2} d f h$ & $b^{3} c e i$ & $b^{5} g^{2}$ & $b^{5} c f^{2}$ & $b^{2} c^{6} d$ & $b^{12} c d$ \\
\hline$b^{4} c^{3} d^{2}$ & $\mathrm{fm}$ & $b d^{2} k$ & $b^{2} d g^{2}$ & $b^{3} c f h$ & $b^{4} c^{2} j$ & $b^{5} d^{2} g$ & $b c^{8}$ & $b^{11} c^{3}$ \\
\hline$b^{3} c^{5} d$ & $g l$ & $b d e j$ & $b^{2} e^{2} h$ & $b^{3} c g^{2}$ & $b^{4} c d i$ & $b^{5}$ def & $b^{9} i$ & $b^{14} d$ \\
\hline$b^{2} c^{7}$ & $h k$ & $b d f i$ & $b^{2} e f g$ & $b^{3} d^{2} i$ & $b^{4} c e h$ & $b^{5} e^{3}$ & $b^{8} c h$ & $b^{13} c^{2}$ \\
\hline$b^{9} h$ & $i j$ & $b d g h$ & $b^{2} f^{3}$ & $b^{3} d e h$ & $b^{4} c f g$ & $b^{4} c^{3} h$ & $b^{8} d g$ & $b^{15} c$ \\
\hline$b^{8} \mathrm{cg}$ & $b^{2} p$ & $b e^{2} i$ & $b c^{3} k$ & $b^{3} d f g$ & $b^{4} d^{2} h$ & $b^{4} c^{2} d g$ & $b^{8} e f$ & $b^{17}$ \\
\hline$b^{8} d f$ & $b c o$ & befh & $b c^{2} d j$ & $b^{3} e^{2} g$ & $b^{4} \mathrm{deg}$ & $b^{4} c^{2} e f$ & $b^{7} c^{2} g$ & \\
\hline$b^{8} e^{2}$ & $b d n$ & $b e g^{2}$ & $b c^{2} e i$ & $b^{3} e f^{2}$ & $b^{4} d f^{2}$ & $b^{4} c d^{2} f$ & $b^{7} c d f$ & 18 \\
\hline$b^{7} c^{2} f$ & bem & $b f^{2} g$ & $b c^{2} f h$ & $b^{2} c^{3} j$ & $b^{4} e^{2} f$ & $b^{4} c d e^{2}$ & $b^{7} c e^{2}$ & 385 \\
\hline$b^{7} c d e$ & $b f l$ & $c^{3} l$ & $b c^{2} g^{2}$ & $b^{2} c^{2} d i$ & $b^{3} c^{3} i$ & $b^{4} d^{3} e$ & $b^{7} d^{2} e$ & $s$ \\
\hline$b^{7} d^{3}$ & $b g k$ & $c^{2} d k$ & $b c d^{2} i$ & $b^{2} c^{2} e h$ & $b^{3} c^{2} d h$ & $b^{3} c^{4} g$ & $b^{6} c^{3} f$ & $b r$ \\
\hline$b^{6} c^{3} e$ & $b h j$ & $c^{2} e j$ & bcdeh & $b^{2} c^{2} f g$ & $b^{3} c^{2} e g$ & $b^{3} c^{3} d f$ & $b^{6} c^{2} d e$ & $c q$ \\
\hline$b^{6} c^{2} d^{2}$ & $b i^{2}$ & $c^{2} f i$ & $b c d f g$ & $b^{2} c d^{2} h$ & $b^{3} c^{2} f^{2}$ & $b^{3} c^{3} e^{2}$ & $b^{6} c d^{3}$ & $d p$ \\
\hline$b^{5} c^{4} d$ & $c^{2} n$ & $c^{2} g h$ & $b c e^{2} g$ & $b^{2} c d e g$ & $b^{3} c d^{2} g$ & $b^{3} c^{2} d^{2} e$ & $b^{5} c^{4} e$ & eo \\
\hline$b^{4} c^{6}$ & $c d m$ & $c d^{2} j$ & $b c e f^{2}$ & $b^{2} c d f^{2}$ & $b^{3} c d e f$ & $b^{3} c d^{4}$ & $b^{5} c^{3} d^{2}$ & $f n$ \\
\hline$b^{10} g$ & cel & $c d e i$ & $b d^{3} h$ & $b^{2} c e^{2} f$ & $b^{3} c e^{3}$ & $b^{2} c^{5} f$ & $b^{4} c^{5} d$ & $g m$ \\
\hline$b^{9} c f$ & $c f k$ & $c d f h$ & $b d^{2} e g$ & $b^{2} d^{3} g$ & $b^{3} d^{3} f$ & $b^{2} c^{4} d e$ & $b^{3} c^{7}$ & $h l$ \\
\hline$b^{9} \mathrm{de}$ & $c g j$ & $c d g^{2}$ & $b d^{2} f^{2}$ & $b^{2} d^{2} e f$ & $b^{3} d^{2} e^{2}$ & $b^{2} c^{3} d^{3}$ & $b^{10} h$ & $i k$ \\
\hline$b^{8} c^{2} e$ & $c h i$ & $c e^{2} h$ & $b d e^{2} f$ & $b^{2} d e^{3}$ & $b^{2} c^{4} h$ & $b c^{6} e$ & $b^{9} \mathrm{cg}$ & $j^{2}$ \\
\hline$b^{8} c d^{2}$ & $d^{2} l$ & $c e f g$ & $b e^{4}$ & $b c^{4} i$ & $b^{2} c^{3} d g$ & $b c^{5} d^{2}$ & $b^{9} d f$ & $b^{2} q$ \\
\hline$b^{7} c^{3} d$ & dek & $c f^{3}$ & $c^{4} j$ & $b c^{3} d h$ & $b^{2} c^{3} e f$ & $c^{7} d$ & $b^{9} e^{2}$ & $b c p$ \\
\hline$b^{6} c^{5}$ & $d f j$ & $d^{3} i$ & $c^{3} d i$ & $b c^{3} e g$ & $b^{2} c^{2} d^{2} f$ & $b^{8} j$ & $b^{8} c^{2} f$ & $b d o$ \\
\hline$b^{11} f$ & $d g i$ & $d^{2} e h$ & $c^{3} e h$ & $b c^{3} f^{2}$ & $b^{2} c^{2} d e^{2}$ & $b^{7} c i$ & $b^{8} c d e$ & ben \\
\hline$b^{10} c e$ & $d h^{2}$ & $d^{2} f g$ & $c^{3} f g$ & $b c^{2} d^{2} g$ & $b^{2} c d^{3} e$ & $b^{7} d h$ & $b^{8} d^{3}$ & $b f m$ \\
\hline$b^{10} d^{2}$ & $e^{2} j$ & $d e^{2} g$ & $c^{2} d^{2} h$ & $b c^{2} d e f$ & $b^{2} d^{5}$ & $b^{7} e g$ & $b^{7} c^{3} e$ & $b g l$ \\
\hline
\end{tabular}


The Partition Table, 0 to 18 (continued).

\begin{tabular}{|c|c|c|c|c|c|c|c|c|}
\hline 18 & 18 & 18 & 18 & 18 & 18 & 18 & 18 & 18 \\
\hline$b h k$ & bef $i$ & $b^{2} d e j$ & $d^{3} e f$ & $b c^{2} \mathrm{deg}$ & $b^{3} c d f^{2}$ & $b^{4} c^{3} i$ & $b^{5} c d^{2} f$ & $b^{8} c^{2} g$ \\
\hline$b \ddot{i j}$ & begh & $b^{2} d f i$ & $d^{2} e^{3}$ & $b c^{2} d f^{2}$ & $b^{3} c e^{2} f$ & $b^{4} c^{2} d h$ & $b^{5} c d e^{2}$ & $b^{8} c d f$ \\
\hline$c^{2} o$ & $b f^{2} h$ & $b^{2} d g h$ & $b^{5} n$ & $b c^{2} e^{2} f$ & $b^{3} d^{3} g$ & $b^{4} c^{2} e g$ & $b^{5} d^{3} e$ & $b^{8} c e^{2}$ \\
\hline$c d n$ & $b f g^{2}$ & $b^{2} e^{2} i$ & $b^{4} \mathrm{~cm}$ & $b c d^{3} g$ & $b^{3} d^{2} e f$ & $b^{4} c^{2} f^{2}$ & $b^{4} c^{4} g$ & $b^{8} d^{2} e$ \\
\hline cem & $c^{3} m$ & $b^{2} e f h$ & $b^{4} d l$ & $b c d^{2} e f$ & $b^{3} d e^{3}$ & $b^{4} c d^{2} g$ & $b^{4} c^{3} d f$ & $b^{7} c^{3} f$ \\
\hline$c f l$ & $c^{2} d l$ & $b^{2} e g^{2}$ & $b^{4} e k$ & $b c d e^{3}$ & $b^{2} c^{4} i$ & $b^{4} c d e f$ & $b^{4} c^{3} e^{2}$ & $b^{7} c^{2} d e$ \\
\hline$c g k$ & $c^{2} e k$ & $b^{2} f^{2} g$ & $b^{4} f j$ & $b d^{4} f$ & $b^{2} c^{3} d h$ & $b^{4} c e^{3}$ & $b^{4} c^{2} d^{2} e$ & $b^{7} c d^{3}$ \\
\hline$c h j$ & $c^{2} f j$ & $b c^{3} l$ & $b^{4} g i$ & $b d^{3} e^{2}$ & $b^{2} c^{3} e g$ & $b^{4} d^{3} f$ & $b^{4} c d^{4}$ & $b^{6} c^{4} e$ \\
\hline$c i^{2}$ & $c^{2} g i$ & $b c^{2} d k$ & $b^{4} h^{2}$ & $c^{5} \boldsymbol{i}$ & $b^{2} c^{3} f^{2}$ & $b^{4} d^{2} e^{2}$ & $b^{3} c^{5} f$ & $b^{6} c^{3} d^{2}$ \\
\hline$d^{2} m$ & $c^{2} h^{2}$ & $b c^{2} e j$ & $b^{3} c^{2} l$ & $c^{4} d h$ & $b^{2} c^{2} d^{2} g$ & $b^{3} c^{4} h$ & $b^{3} c^{4} d e$ & $b^{5} c^{5} d$ \\
\hline del & $c d^{2} k$ & $b c^{2} f i$ & $b^{3} c d k$ & $c^{4} e g$ & $b^{2} c^{2} d e f$ & $b^{3} c^{3} d g$ & $b^{3} c^{3} d^{3}$ & $b^{4} c^{7}$ \\
\hline$d f k$ & $c d e j$ & $b c^{2} g h$ & $b^{3} c e j$ & $c^{4} f^{2}$ & $b^{2} c^{2} e^{3}$ & $b^{3} c^{3} e f$ & $b^{2} c^{6} e$ & $b^{11} h$ \\
\hline$d g j$ & $c d f i$ & $b c d^{2} j$ & $b^{3} c f i$ & $c^{3} d^{2} g$ & $b^{2} c d^{3} f$ & $b^{3} c^{2} d^{2} f$ & $b^{2} c^{5} d^{2}$ & $b^{10} c g$ \\
\hline$d h i$ & $c d g h$ & bcdei & $b^{3} c g h$ & $c^{3} d e f$ & $b^{2} c d^{2} e^{2}$ & $b^{3} c^{2} d e^{2}$ & $b c^{7} d$ & $b^{10} d f$ \\
\hline$e^{2} k$ & $c e^{2} i$ & bcdfh & $b^{3} d^{2} j$ & $c^{3} e^{3}$ & $b^{2} d^{4} e$ & $b^{3} c d^{3} e$ & $c^{9}$ & $b^{10} e^{2}$ \\
\hline$e f j$ & cefh & $b c d g^{2}$ & $b^{3} d e i$ & $c^{2} d^{3} f$ & $b c^{5} h$ & $b^{3} d^{5}$ & $b^{9} j$ & $b^{9} c^{2} f$ \\
\hline$e g i$ & $c e g^{2}$ & $b c e^{2} h$ & $b^{3} d f h$ & $c^{2} d^{2} e^{2}$ & $b c^{4} d g$ & $b^{2} c^{5} g$ & $b^{8} c i$ & $b^{9} c d e$ \\
\hline$e h^{2}$ & $c f^{2} g$ & bcefg & $b^{3} d g^{2}$ & $c d^{4} e$ & $b c^{4} e f$ & $b^{2} c^{4} d f$ & $b^{8} d h$ & $b^{9} d^{3}$ \\
\hline$f^{2} i$ & $d^{3} j$ & $b c f^{3}$ & $b^{3} e^{2} h$ & $d^{6}$ & $b c^{3} d^{2} f$ & $b^{2} c^{4} e^{2}$ & $b^{8} e g$ & $b^{8} c^{3} e$ \\
\hline$f g h$ & $d^{2} e i$ & $b d^{3} i$ & $b^{3} e f g$ & $b^{6} m$ & $b c^{3} d e^{2}$ & $b^{2} c^{3} d^{2} e$ & $b^{8} f^{2}$ & $b^{8} c^{2} d^{2}$ \\
\hline$g^{3}$ & $d^{2} f h$ & $b d^{2} e h$ & $b^{3} f^{3}$ & $b^{5} c l$ & $b c^{2} d^{3} e$ & $b^{2} c^{2} d^{4}$ & $b^{7} c^{2} h$ & $b^{7} c^{4} d$ \\
\hline$b^{3} p$ & $d^{2} g^{2}$ & $b d^{2} f g$ & $b^{2} c^{3} k$ & $b^{5} d k$ & $b c d^{5}$ & $b c^{6} f$ & $b^{7} c d g$ & $b^{6} c^{6}$ \\
\hline$b^{2} c o$ & $d e^{2} h$ & $b d e^{2} g$ & $b^{2} c^{2} d j$ & $b^{5} e j$ & $c^{6} g$ & $b c^{5} d e$ & $b^{7} c e f$ & $b^{12} g$ \\
\hline$b^{2} d n$ & defg & $b d e f^{2}$ & $b^{2} c^{2} e i$ & $b^{5} f i$ & $c^{5} d f$ & $b c^{4} d^{3}$ & $b^{7} d^{2} f$ & $b^{11} c f$ \\
\hline$b^{2} \mathrm{em}$ & $d f^{3}$ & $b e^{3} f$ & $b^{2} c^{2} f h$ & $b^{5} g h$ & $c^{5} e^{2}$ & $c^{7} e$ & $b^{7} d e^{2}$ & $b^{11} d e$ \\
\hline$b^{2} f l$ & $e^{3} g$ & $c^{4} k$ & $b^{2} c^{2} g^{2}$ & $b^{4} c^{2} k$ & $c^{4} d^{2} e$ & $c^{6} d^{2}$ & $b^{6} c^{3} g$ & $b^{10} c^{2} e$ \\
\hline$b^{2} g k$ & $e^{2} f^{2}$ & $c^{3} d j$ & $b^{2} c d^{2} i$ & $b^{4} c d j$ & $c^{3} d^{4}$ & $b^{8} k$ & $b^{6} c^{2} d f$ & $b^{10} c d^{2}$ \\
\hline$b^{2} h j$ & $b^{4} O$ & $c^{3} e i$ & $b^{2} c d e h$ & $b^{4} c e i$ & $b^{7} l$ & $b^{7} c j$ & $b^{6} c^{2} e^{2}$ & $b^{9} c^{3} d$ \\
\hline$b^{2} i^{2}$ & $b^{3} c n$ & $c^{3} f h$ & $b^{2} c d f g$ & $b^{4} c f h$ & $b^{6} c k$ & $b^{7} d i$ & $b^{6} c d^{2} e$ & $b^{8} c^{5}$ \\
\hline$b c^{2} n$ & $b^{3} d m$ & $c^{3} g^{2}$ & $b^{2} c e^{2} g$ & $b^{4} c g^{2}$ & $b^{6} d j$ & $b^{7} e h$ & $b^{6} d^{4}$ & $b^{13} f$ \\
\hline$b c d m$ & $b^{3} e l$ & $c^{2} d^{2} i$ & $b^{2} c e f^{2}$ & $b^{4} d^{2} i$ & $b^{6} e i$ & $b^{7} f g$ & $b^{5} c^{4} f$ & $b^{12} c e$ \\
\hline bcel & $b^{3} f k$ & $c^{2} d e h$ & $b^{2} d^{3} h$ & $b^{4} d e h$ & $b^{6} f h$ & $b^{6} c^{2} i$ & $b^{5} c^{3} d e$ & $b^{12} d^{2}$ \\
\hline$b c f k$ & $b^{3} g j$ & $c^{2} d f g$ & $b^{2} d^{2} e g$ & $b^{4} d f g$ & $b^{6} g^{2}$ & $b^{6} c d h$ & $b^{5} c^{2} d^{3}$ & $b^{11} c^{2} d$ \\
\hline$b c g j$ & $b^{3} h i$ & $c^{2} e^{2} g$ & $b^{2} d^{2} f^{2}$ & $b^{4} e^{2} g$ & $b^{5} c^{2} j$ & $b^{6} c e g$ & $b^{4} c^{5} e$ & $b^{10} c^{4}$ \\
\hline$b c h i$ & $b^{2} c^{2} m$ & $c^{2} e f^{2}$ & $b^{2} d e^{2} f$ & $b^{4} e f^{2}$ & $b^{5} c d i$ & $b^{6} c f^{2}$ & $b^{4} c^{4} d^{2}$ & $b^{14} e$ \\
\hline$b d^{2} l$ & $b^{2} c d l$ & $c d^{3} h$ & $b^{2} e^{4}$ & $b^{3} c^{3} j$ & $b^{5} c e h$ & $b^{6} d^{2} g$ & $b^{3} c^{6} d$ & $b^{13} c d$ \\
\hline bdek & $b^{2} c e k$ & $c d^{2} e g$ & $b c^{4} j$ & $b^{3} c^{2} d i$ & $b^{5} c f g$ & $b^{6} d e f$ & $b^{2} c^{8}$ & $b^{12} c^{3}$ \\
\hline$b d f j$ & $b^{2} c f j$ & $c d^{2} f^{2}$ & $b c^{3} d i$ & $b^{3} c^{2} e h$ & $b^{5} d^{2} h$ & $b^{6} e^{3}$ & $b^{10} i$ & $b^{15} d$ \\
\hline$b d g i$ & $b^{2} c g i$ & $c d e^{2} f$ & $b c^{3} e h$ & $b^{3} c^{2} f g$ & $b^{5} \mathrm{deg}$ & $b^{5} c^{3} h$ & $b^{9} c h$ & $b^{14} c^{2}$ \\
\hline$b d h^{2}$ & $b^{2} c h^{2}$ & $c e^{4}$ & $b c^{3} f g$ & $b^{3} c d^{2} h$ & $b^{5} d f^{2}$ & $b^{5} c^{2} d g$ & $b^{9} d g$ & $b^{16} c$ \\
\hline$b e^{2} j$ & $b^{2} d^{2} k$ & $d^{4} g$ & $b c^{2} d^{2} h$ & $b^{3} c d e g$ & $b^{5} e^{2} f$ & $b^{5} c^{2} e f$ & $b^{9} e f$ & $b^{18}$ \\
\hline
\end{tabular}

